\title{
The Role of Flare Stars in Cosmic-ray Origin
}

\author{
Maurice M. Shapiro \\ University of Maryland, College Park, MD, USA*
}

\begin{abstract}
Supernovae and their expanding shock fronts are evidently the main agents of cosmic-ray acceleration. The thermal gas in the interstellar medium has been regarded as the reservoir of seed particles destined to become cosmicray nuclei. This assumption is, however, at variance with the source composition of galactic cosmic rays. In an alternative hypothesis, the seed particles are injected into the interstellar material as suprathermal seed ions, and it has been surmised that flare stars provide the initial boost. We find that the dMe and dKe stars are probably the principal sources of cosmic-ray seed particles. Most stars in the Galaxy are red dwarfs and many of these flares much more powerfully and frequently than solar flares. Augmenting the optical data, recent X-ray and far-ultraviolet observations now permit a better estimate of the energy budget. Altogether, $\mathrm{dMe}$ and $\mathrm{dKe}$ stars seem to be the most promising class of cosmic-ray injectors.
\end{abstract}

\section{Introduction}

According to well-founded theory, most of the galactic cosmic rays (GCR) are accelerated to their high energies by shock waves in the interstellar medium (ISM). This theory has generally assumed that the shock fronts, generated mainly by supernova explosions, pick up the particles directly from the thermal pool of ions in the ISM gas. This seems to imply that no special injection of suprathermal seed particles is required prior to their main acceleration. There are compelling reasons to question this view. These are based on a comparison of the element abundances in the ISM gas with the source composition inferred from (a) observations of the "arriving" GCR composition in the vicinity of Earth, and (b) analysis of $C R$ propagation in the Galaxy.

We represent an alternative model - one that yields better agreement with observations (Shapiro 1987, 1990). It invokes the injection of energetic seed ions from stellar flares, and especially those of $\mathrm{dMe}$ and $\mathrm{dKe}$ stars, as a precursor to the main accelerating process. The model is supported by examination of the relevant physical parameters.

* Address for correspondence: 205 Yoakum Pkwy 2-1514, Alexandria, VA 22304 
In Sect. 2, we summarize some of the earlier ideas on cosmic ray origin (Fermi 1949, Ginzburg 1951, Shklovsky 1953, Shapiro 1962, Ginzburg and Syrovatsky 1964). Then we discuss the more recent theory of shock-wave acceleration. Sect. 3 explains why injection of suprathermal seed particles is required. Sect. 4 describes the hypothesis of injection from stellar flares, comparing these to solar flares. It focuses on the $\mathrm{dMe}$ and $\mathrm{dKe}$ dwarfs, as these are likely to be the dominant contributors of energetic seed ions for shock acceleration to GCR.

Sect. 5 discusses the energy budget of the cosmic-ray reservoir in the Galaxy, and the available supply of stellar seed ions to feed shock acceleration. It attempts to evaluate the various parameters that determine the adequacy of the particle-energy supply from stellar flares. Sect. 6 deals with the question as to whether emitted particles will meet a supernova shock. Sect. 7 considers the possible role of other types of flare stars as injectors. It also mentions the possible contribution of stellar winds. Finally, Sect. 8 discusses some related issues, and summarizes our conclusions.

\section{Magnetohydrodynamic theories of cosmic-ray origin}

Fermi's seminal proposal for cosmic-ray acceleration (Fermi 1949, Chandrasekhar \& Fermi 1953) were based partly on principles of magnetohydrodynamics developed by Alfvén (1981) and others. In Fermi's first scheme - the statistical mechanism - acceleration is produced by collisions of particles with magnetized clouds in the ISM. A notable success of this "second-order theory" is that it gave a power-law energy spectrum. It proved, however, to be too slow; moreover, it did not work for heavy CR nuclei. Fermi then developed another theory involving special magnetic-field configurations in the ISM; these resembled the mirror-machines subsequently developed for controlled fusion devices.

Central to Fermi's concepts, as well as to recent schemers of shock acceleration, was the mechanism of energization by particle collisions with moving magnetic "walls" that reflect and accelerate ions. Hence subsequent theories (e.g. Krymsky 1977, Bell 1978, Eichler 1979, Axford et al. 1977, Blandford \& Ostriker 1978 ) are still called "Fermi acceleration" even though he did not invoke shocks.

Given a strong shock front that compresses the gas in its path, and the diffusion of particles between scattering centers in the ISM, ions are repeatedly reflected back and forth, gaining energy at each step. The resulting energy spectrum conforms to a power law $E^{-2}$, consistent with the observed CR spectrum, after correction for the preferential escape of particles with the higher rigidities (Cesarsky 1983). A high-energy cutoff $\left(\approx 10^{14} \mathrm{eV}\right)$ is predicted for shock acceleration in the Galaxy (Lagage 1983).

It was formerly supposed that $\mathrm{CR}$ are literally born in processes of stellar collapse that produce supernovae (SN), i.e. that the CR precursor particles are picked up from material ejected into the SN remnant (Ginzburg 1951, Shapiro 1962, Ginzburg \& Syrovatsky 1964). Today it still seems possible that some relativistic nuclei and electrons could arise in this way. (Here we shall be dealing with the CR nuclei.) For nearly two decades acceleration theory has continued 
to regard SN explosions as the principal "machine", i.e. as the energy source, through the action of shock fronts at the leading edge of expanding SN shells. However, in current models for the origin of the preponderant bulk of cosmicrays (say, up to $10^{13} \mathrm{eV} / \mathrm{amu}^{2}$ ) the atomic nuclei that are ultimately promoted to $\mathrm{CR}$ are assumed to reside in the ISM, and there to be accelerated by the shocks they encounter ${ }^{3}$.

In Sect. 1 we mentioned that in the theories of acceleration by shock waves it has been tacitly (if not always explicitly) assumed that the seed-ions reside in the background of thermal gas in the ISM, i.e., that they are directly picked up from the thermal pool by the shock fronts. While this possibility cannot yet be ruled out, we are here arguing that injection is ordinarily required for the acceleration of the GCR. As will be seen, we invoke a promising source of suprathermal ions for the seed particles.

\section{The observed composition of GCR constrains the source of seed ions}

The source composition of the GCR is well correlated with the preferential selection - prior to injection or acceleration - of those heavy ions with relatively lower $(<10 \mathrm{eV})$ first ionization potentials (FIP). This mechanism works well at temperatures in the atmospheres of $M$ and $K$ dwarfs. The ions are transported to the corona, where they are energized to $\mathrm{MeV}$ and $\mathrm{keV}$ energies in flares. Selection by FIP favors a scenario of origin of the CR progenitor ions in stellar envelopes, and their subsequent injection into the ISM, rather than direct shock acceleration of thermal particles in the ambient ISM. Most of the volume of the ISM is in the hot phase, at $\approx 10^{6} \mathrm{~K}-$ a temperature too high for the observed FIP selection.

Finally we note that while shocks can energize thermal ions such as those in the ISM, the efficiency for picking up nuclei with $\mathrm{MeV}$ energies is greater than that for thermal ones. This, by itself, is not a cogent argument for injection. It is rather the compositional constraints cited above that indicate a need to inject seed particles from another source.

\section{Injection from stellar flares}

Solar flares produce energetic particles that have, in fact been called solar cosmic rays. Here we shall refer to them as solar energetic particles (SEP); they differ significantly from the GCR, especially in their energy spectra, although the

${ }^{2}$ It is noteworthy that $<10^{-4}$ of the CR nuclei have energies exceeding $10^{12} \mathrm{eV} / \mathrm{amu}$. The electron component, which is not discussed here, contributes less than $2 \%$ of the arriving CR.

3 Of course, it is recognized that most of the heavy atoms ("metals") originally came into the ISM, and thence into second-generation stars, from the debris of SN explosions 
typical SEP composition has much in common with that of the CR sources. The latter have a relatively flat spectrum, whereas the SEP spectra are very steep, with a preponderance of $\mathrm{MeV}$ particles. It has long been known that late-type main sequence stars, notably $M$ and $K$ dwarfs, display prolific flaring activity. In particular, the $\mathrm{dMe}$ and the $\mathrm{dKe}$ stars, so designated because of their prominent emission lines, have frequent flares (Gershberg 1988, Shakhovskaya 1995). The exceptionally strong flares that occur only once in several years in the Sun seem commonplace among $\mathrm{dMe}$ and $\mathrm{dKe}$ stars. We shall usually be referring to these dwarfs when we use the terms "flare stars" or "stellar flares", although there are other active stars - far less numerous - that show conspicuous flaring activity. As to solar flares, it is noteworthy - and hardly the result of pure coincidence - that their SEP exhibit the same FIP selection as described in Sect. 3 for the GCR (Meyer 1985).

\section{Energy budget for injection}

In assessing the adequacy of stellar flares as injectors, we compare the power density $W-\mathrm{i}$ available for injection from flares, with the power density $W_{\mathrm{Cr}}$ of the GCR. In so doing, we confront the following questions: What is the rate $W_{\mathrm{em}}$ of energy emission in electromagnetic radiations ( $\mathrm{UV}, \mathrm{X}$-ray, and visible light) per unit volume of the ISM? How much of the flare energy is likely to go into the production of energetic nuclei? What fraction $f_{\mathrm{i}}$ of these escape into the ISM? If $\mathrm{SN}$ shocks (and other shocks) boost the ions to $\mathrm{CR}$, does the rate of supply of injected particles suffice to meet the requirements imposed by the observed CR energy density, lifetime, and mean particle energy? Further, once the ions escape from a flare, what is their potential slowing-down time $t$ ? Within that time, is it likely that the particle will encounter at least one shock front in the ISM?

Many of these questions do not yet have firm answers; only rough estimates are available for some of the parameters, while reasonable limits can be assigned to others. Despite disparities in published data (a dilemma not unknown to astrophysics) we try, by adopting conservative values and/or lower limits, to arrive at tenable conclusions.

Let us estimate the power density $W_{\mathrm{i}}$ introduced above. For the average flare, we define:

$E_{\text {em }}$ is the energy in the form of electromagnetic radiation (visible $+U V+$ $\mathrm{X}$-ray),

$\nu_{\mathrm{f}}$ is the corresponding flare frequency,

$E_{\mathrm{p}}$ is the flare energy that goes into ion production,

$E_{\mathrm{i}}$ is the energy in ions that escape from a flare,

$f_{\mathrm{i}}$ is the ratio $E_{\mathrm{i}} / E_{\mathrm{p}}$.

Observations and theory suggest that particle acceleration is intimately tied to the emission of radiation. Hence, lacking definitive data, we assume in our model that

$$
E_{\mathrm{p}} \approx E_{\mathrm{em}}
$$


Now let $W_{\mathrm{p}}$ be the power density for the production of fast ions in flares. Let $W_{\mathrm{i}}$ be the power density in fast ions escaping from flares, and $n_{\mathrm{d}}$ be the mean number density of dMe and dKe stars in the Galaxy.

Based on data in the flare literature (some of which allow considerable leeway), we provisionally adopt values of the parameters that probably yield an underestimate ${ }^{4}$ of $W_{i}$. These values are based in part on the extensive flare observations of Gershberg (1988) and Shakhovskaya (1995):

$n_{\mathrm{d}}=0.04 \mathrm{pc}^{-3}$,

$E_{\text {em }}=10^{34} \mathrm{erg}$,

$\nu_{\mathrm{f}}=(100 \mathrm{~h})^{-1}=\left(3.6 \times 10^{5} \mathrm{~s}\right)^{-1}$.

$f_{\mathrm{i}}=0.15$.

The power density in emitted energetic ions is:

$$
W_{\mathrm{i}}=n_{\mathrm{d}} E_{\mathrm{em}} \nu_{\mathrm{f}} f_{\mathrm{i}}=1.7 \cdot 10^{26} \mathrm{erg} \mathrm{pc}^{-3} \mathrm{~s}^{-1} .
$$

Let $W_{\text {cr }}$ be the power density in GCR.

$$
\begin{aligned}
W_{\mathrm{CR}} & =\text { energy density } / \text { lifetime } \\
& =1 \mathrm{eV} \mathrm{cm}-3 / 1.3 \cdot 10^{7} \mathrm{y} \\
& =1.15 \cdot 10^{29} \mathrm{erg} \mathrm{pc}^{-3} \mathrm{~s}^{-1}
\end{aligned}
$$

Define $\Gamma \equiv W_{\mathrm{i}} / W_{\mathrm{CR}}$. Then,

$$
\Gamma \approx 1.5 \cdot 10^{-3}
$$

Thus, the power required - and available for injection - is a small fraction of that needed for replenishment of the GCR as these escape from the CR reservoir in the Galaxy.

\section{Will the emitted particles meet a shock?}

Next we estimate the probability $P$ that the ions will meet a strong shock, i.e. one sufficiently powerful to accelerate them to $C R$ energies. This depends on the time $t_{\mathrm{S}}$ available for such encounters, and on the fraction of the Galactic volume that contains the expanding envelopes of SN remnants.

In evaluating $t_{\mathrm{S}}$, we use the range-energy tables of Northcliff \& Schilling (1970). First, however, we note that the mean energy of the emitted ions is obtainable from the value of $\Gamma$ in Eq. 4 . Let $w_{\mathrm{i}}$ be the mean particle energy of the seed ions, and $w_{\text {cr }}$ be the mean energy of a GCR nucleus, $\approx 6 \mathrm{GeV}$. Then $w_{\mathrm{i}}=\Gamma w_{\mathrm{CR}}$. With Eq. $4, w_{\mathrm{i}} \approx 9 \mathrm{MeV}$. Further, to compute the range, we adopt a mean value of $0.1 \mathrm{H}$ atom $\mathrm{cm}^{3}$ for the number density in the ISM, most of which consists of rarefied gas in the hot phase. The slowing-down time $t_{\mathrm{s}}$ for a $9 \mathrm{MeV}$ ion will then be $\approx 12$ million years - about the same as the lifetime of the CR against escape from the Galaxy.

\footnotetext{
${ }^{4}$ In Sect. 8 we refer to an independent study which suggests that our provisional estimate of $W_{i}$ is a lower limit.
} 
With a SN rate of $(50 \mathrm{y})^{-1}$ in the Galaxy, there will be $2.4 \times 10^{5} \mathrm{SN}$ outbursts in $1.2 \cdot 10^{7}$ years. The total volume $V_{8 n}$ filled by their remnants, each within a radial distance $r_{\mathrm{g}}$ of $70 \mathrm{pc}$ is $4.3 \cdot 10^{11} \mathrm{pc}^{3}$. (At $r>r_{\mathrm{s}}$, the shocks become weak).

To evaluate $P$, we estimate the volume $V_{g}$ of the Galaxy with a disk thickness of $10^{3} \mathrm{pc}$. This yields $V_{\mathrm{g}} \approx 5 \cdot 10^{11} \mathrm{pc}^{3}$. Within the uncertainties in the values of $V_{\mathrm{sn}}$ and $V_{\mathrm{g}}, P$ is of the order unity. Thus it appears that the seed ions are likely to meet a shock.

\section{Other flare stars; stellar winds}

We have devoted this paper mainly to $\mathrm{CR}$ injection from dMe and dKe stars, owing to their overwhelming numbers and to the frequency of their intense flares. Other flaring stars are widely discussed (e.g. Haisch \& Rodonò 1989, Haisch et al. 1991, Wallerstein 1990), and their features are described elsewhere in these Proceedings. Their numbers are small compared with those of red dwarfs: yet they may add to the pool of seed particles, provided their atmospheres are conductive to ion selection yielding the FIP effect. We make no effort here at quantitative estimates of their net effect.

Stellar winds consist of much slower particles than the ions from stellar flares. Nevertheless, it seems possible that, under appropriate conditions, they might also provide some CR seed particles. This topic is beyond the scope of the present discussion.

Probably we have not established a conclusive argument for the adequacy of the red dwarf flares as the principal injectors of GCR (see remarks on the uncertainties below). It should be noted, however, that the case for requiring a suitable source of injected particles is compelling, as shown in Sect. 3.

\section{Discussion and summary}

The idea that there might be a connection between CR and stellar emissions is not new ${ }^{5}$ (Unsöld 1957, Edwards \& McQueen 1977). That flares on $M$ and $K$ stars might directly produce the lower-energy $\left(<10^{9} \mathrm{eV}\right) \mathrm{CR}$ was proposed by Lovell (1974). Later, Mullan (1979) concluded that flare stars cannot generate the total CR energy density in the Galaxy. Our analysis supports his result. However, this conclusion by no means precludes the more modest role that flare stars seem to play as injectors. Based on early X-ray observations, Gorenstein ( 1981) favored a hypothesis similar to Lovell's. Arguments were adduced by Cassé ((183) and Meyer 91985) to show that the nature of the CGR source composition is inconsistent with the direct acceleration of thermal ISM material. As to the stellar injection of GCR, the first attempt to go beyond speculation, and to treat the problem quantitatively, was made by Shapiro (1990). The present paper is an updated version, and a fuller treatment of that earlier work.

\footnotetext{
${ }^{5}$ Today it seems curious that the Sun itself was once regarded by a few eminent physicists as the source of CR.
} 
We have avoided assigning figures of merit to the parametric values used here. In the present state of our knowledge of M-dwarf flares, estimates of error for most of the parameters would be rather subjective. As an example of the difficulties in observing and measuring the properties of faint stars like red dwarfs, consider the value assigned to the electromagnetic energy emission from $\mathrm{dMe}$ and $\mathrm{dKe}$ stars. The value in optical emission is based mainly on extensive observations (Gershberg 1988, Shakhovskaya 1995). However, our tentative estimate that UV and X-ray emission each contributes energy comparable to that in the optical channel is based on a limited number of simultaneous detections of flares at the various wavelengths. Clearly the simultaneous observations from space telescopes required for the $\mathrm{X}$-ray and UV emissions from flares occurring at unpredictable times on faint stars, present logistical problems; the data are accumulating slowly. Moreover, our assumption (Eq. 1) that the energy going into production of flare particles is comparable to that in electromagnetic radiation is based largely on theoretical considerations rather than on available data. Our value for $f_{\mathrm{i}}$, the fraction of ions that escape from a flare is perforce very uncertain. The more reliable figures we have used are those for the GCR, e.g., their energies and lifetimes. At worst, our numerical estimates may be subject to Ginzburg's famous "law of astrophysics", i.,e. $1 \approx 10$.

On the other hand, in discussing our earlier paper (Shapiro 1990), Matthews et al. (1991) asserted that we had underestimated the power density available in injected ions by a very large factor. They based their opinion on analysis of ROSAT X-ray data. If they are correct, energetic seed ions from flaring reddwarf stars would exceed the requirements for CR injection, irrespective of the uncertainties cited above. It would certainly bolster my belief that the numerical estimates I adopted have been conservative.

In summary, we conclude that $\mathrm{dMe}$ and dKe stars contribute significantly to the injection of GCR. The output of their flares can provide seed particles for acceleration to CR energies, mainly by SN shocks in the ISM. These red dwarfs are unlikely to be the only injectors of the GCR. Yet they seem to be important sources of seed ions since (a) they flare frequently and powerfully, emitting radiations in the various electromagnetic channels; (b) their atmospheres can provide FIP selection that satisfies the GCR source composition; (c) the required energy budget for injection is modest; and (d) once emitted, the ions are likely to meet a strong shock in the ISM. Accordingly, given a need for injection of the GCR, we believe that we have made a plausible case for red dwarf stars as injectors.

Acknowledgments: Among colleagues who have kindly shared their expertise with me are R.E. Gershberg, N. Shakhovskaya, E.N. Parker, M. Rodonò, R. Pallavicini, R. Rosner, and D. Reames. To R. Silberberg I am particularly indebted for helpful discussions.

\section{References}

Alfvén H., 1981, Cosmic Plasmas, Reidel, Dordrecht

Axford W.I., Lear E., Skadron G., 1977, Proc. 15th International Cosmic Ray Conf. 11, p. 132 
Bell A.R., 1978, MNRAS 182, 147

Blandford R.D., Ostriker, J.P., 1978, ApJ 221, L129

Cassé M., 1983, in Composition and Origin of Cosmic Rays, M.M. Shapiro (ed.), Reidel, Dordrecht, p. 193

Cesarsky C.J., 1983, in Composition and Origin of Cosmic Rays, M.M. Shapiro (ed.), Reidel, Dordrecht, p. 161.

Chandrasekhar S., Fermi E., 1953, ApJ 118, 113

Edwards P.J., McQueen M., 1971, Proc. 12th International Cosmic Ray Conf. 1, p. 323

Eichler D. 1979, ApJ 229, 415

Fermi E. 1949, Phys. Rev. 75, 1169

Gershberg R.E., 1988, in Solar and Stellar Flares, Proc. IAU Coll. 104, Kluwer, Dordrecht, p. ...

Ginzburg V.L., 1951, Dokl. Akad. Nauk SSSR 76, 377

Ginzburg V.L., Syrovatsky S.I., 1964, The Origin of Cosmic Rays, Pergamon, London

Gorenstein P., 1981, in Proc. 17th International Cosmic Ray Conf. 12, p. 99

Haisch B.M., Rodonò M. (eds.), 1989, Solar and Stellar Flares, Proc. IAU Coll. 104, Kluwer, Dordrecht

Haisch B.M., Strong K.T., Rodonò M., 1991, ARAA 29, 295

Krymsky G.F., 1977, Dokl. Akad. Nauk SSSR 234, 1306

Lagage P.O., 1983, in Composition and Origin of Cosmic Rays, M.M. Shapiro (ed.), Reidel, Dordrecht, p. 169

Lovell B., 1974, Phil. Trans. Roy. Soc. London A277, 489

Matthew L., Quenby J.J. Sumner T.J., 1991, Proc. 22th International Cosmic Ray Conf. 2, p. 459

Meyer J.-P., 1985, ApJS 57, 173

Mullan D., 1979, ApJ 234, 588

Northcliff L.C., Schilling R.F., 1970, Nuclear Data Tables A7, 233

Shakhovskaya N.I., 1995, these proceedings (and priv. comm.)

Shapiro M.M., 1962, Science 135, 175

Shapiro M.M., 1987, Proc. 20th International Cosmic Ray Conf. 2, p. 260

Shapiro M.M., 1990, Proc. 21th International Cosmic Ray Conf. 4, p. 8

Shklovsky I.S., 1953, Dokl. Akad. Nauk SSSR 90, 983

Unsöld A., 1957, in Radioastronomy, Proc. IAU Symp. 4, H.C. van de Hulst (ed.), Cambridge Univ. Press, Cambridge, p. 242

Wallerstein G. (ed.), 1990, Cool Stars, Stellar Systems, and the Sun, Astr. Soc. Pacific Conf. Series. 